\title{
On the nature of the fine structure in emission spectra of $\mathrm{XeCl}$ laser
}

\author{
I.V. Blonskyy, A.V. Gnatovskyy, N.G. Zubrilin, I.A. Pavlov, M.P. Chernomorets \\ Institute of Physics NAS of Ukraine, 46, prospect Nauky, 03028 Kyiv, Ukraine \\ E-mail: zubrilin@iop.kiev.ua
}

\begin{abstract}
Results of investigation of the fine structure existing in emission spectra of the transverse-discharge pumped $\mathrm{XeCl}$ laser are presented. Influence of selective components of the cavity on the structure of spectrum of induced emission is investigated. Conditions of manifestation in the emission spectra of fine structure caused by the molecules rotation are clarified. Generation frequencies in the region of 0-3 transition are measured.
\end{abstract}

Keywords: laser emission sperctra, fine structure.

Paper received 24.03.04; accepted for publication 21.10.04.

\section{Introduction}

$\mathrm{XeCl}$ laser is, probably, one of the most studied representatives of the devices based upon the monohalogenides of noble gases. In many applications, it is needed a detailed notion of structure of the $\mathrm{XeCl}$ laser emission spectrum and a possibility to control it.

Since the discussion on the nature of the fine structure in the $\mathrm{XeCl}$ laser emission spectrum lasts for a long time [1-5], we conducted a series of experiments targeted at distinguishing between contributions into the emission spectrum from: i) interference in selective components of the cavity and ii) properly the energy structure of $\mathrm{XeCl}$ molecule. In this work, results of our first experiments are presented.

\section{Equipment and technique of investigation}

In this work, an electric-discharge laser with self-acting preionization was used. Its discharge-gap volume was equal to $38 \times 1.5 \times 0.8 \mathrm{~cm}^{3}$ with $1.5 \mathrm{~cm}$ interelectrode spacing. External dielectric mirrors possessing different reflection coefficients served as a cavity. The reflective coating has been put onto one flat of plane-parallel 8-mmwide substrates made of KU-1 type quartz (the coated surface of the substrate was faced towards the interior of the cavity). Substrates of the same type but without coating served as windows of the chamber. "Freon-12" substance $\left(\mathrm{CF}_{2} \mathrm{Cl}_{2}\right)$ was used as a chlorine dopant.

The emission spectrum had been recorded on photo film of RF type by using DFS-8 spec-trograph. With the grating of 1,800 grooves per mm used in the apparatus, the reciprocal linear dispersion was $0.87 \AA / \mathrm{mm}$ in the second order. To identify the wavelengths, the Feemission spectrum of a hollow-cathode lamp was used. The frequencies have been measured by computer treating of the scanned exposed films. The accuracy of the frequency determination is $0.05 \mathrm{~cm}^{-1}$.

\section{Results and discussion}

To reduce the number of components influencing the interference pattern, the following measures were undertaken. The outer surface of the output window was made to be scattering, and external mirrors were absent. Therefore, the cavity consisted of internal surface of the output window and the back window, two surfaces of which could serve as a source of an interference pattern.

The emission spectrum of a laser possessing such a cavity is shown in Fig. 1a. The active mixture inside the chamber was $\mathrm{CF}_{2} \mathrm{Cl}_{2}: \mathrm{Xe}: \mathrm{He}$ in which the components related as 1:45:1500 under a common pressure of $2 \mathrm{~atm}$ $(202,650 \mathrm{~Pa})$. In the spectrum, an interference pattern is observed. The inter-line distance equals to $0.4 \mathrm{~cm}^{-1}$ thus corresponding to width of the window.

The interference pattern was absent (Fig. 1b) in the case in which the cavity was formed by internal surface of the output window and the external 99\%-reflecting mirror placed behind the back window.

The presence of the interference pattern in the former case is explained as follows. One component of the cavity (the back window) is a Fabri-Perot interferometer and, hence, cavity radiation losses are different for different 


\section{I.V. Blonskyy et al.: On the nature of the fine structure in emission spectra of ...}

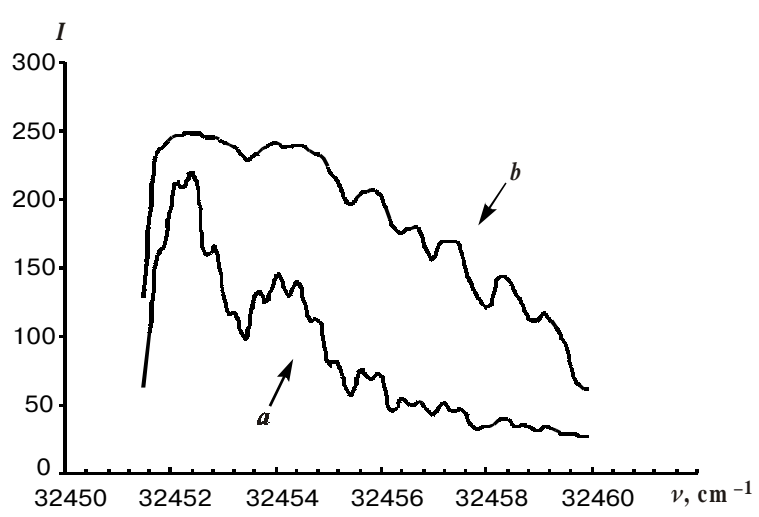

Fig. 1. Emission spectrum of $\mathrm{XeCl}$ laser in the region of $0-2$ transition: $a$-in the presence of interference; $b$ - in the absence of it.

frequencies in accordance with the reflection spectrum of the interferometer. If we place a high-reflective mirror behind this surface, then the cavity losses are determined by this mirror, and the interference pattern disappears. However, it should be taken into account that, when one covers the plane-parallel plate with a mirror layer, the mirror surface and the opposite surface of the substrate also form a Fabri-Perot interferometer with different reflection coefficients of its surfaces.

The reflection spectrum of the mirror that is taken into consideration for the second reflective surface of the substrate is presented in Fig. 2. It is adopted that $R_{1}$ is a reflective surface and $R_{2}=0.04$ - non-covered surface. It is seen that, if high-reflective mirrors are used, the difference between $r_{\max }$ and $r_{\min }$ is negligible. Under $R_{1}=97 \%$, the $r_{\max }-r_{\min }$ value for the reflective surface is $2.4 \%$, and under $R_{1}=99 \%$ it equals to $0.8 \%$. The dependence of $r_{\max }-r_{\min }$ on $R_{1}$ is shown in Fig. 3. $r_{\max }-r_{\min }$ takes its maximum value at $R_{1}$ being about $30 \%$.

From the set forth above, it also follows that a window situated normally to the laser axis does not form the interference pattern in the laser radiation spectrum in the case, when the higher reflectivity mirror is situated be-

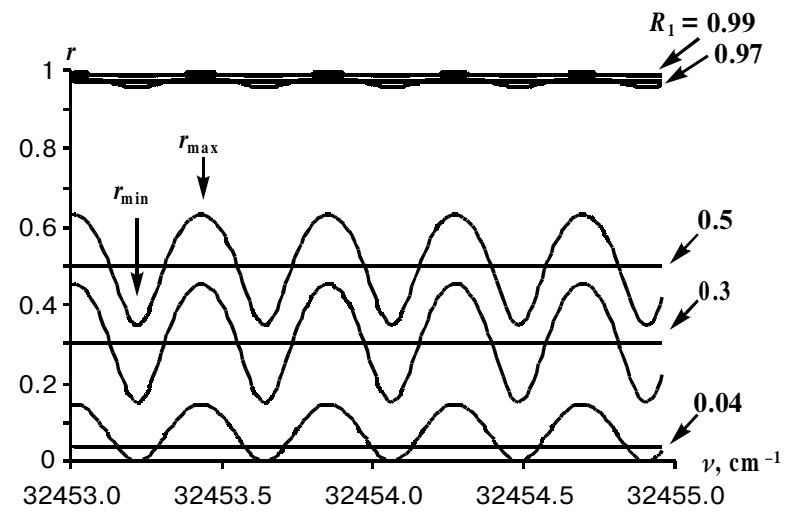

Fig. 2. Dependence of reflection coefficient of the mirror with taking into account the second reflective surface.

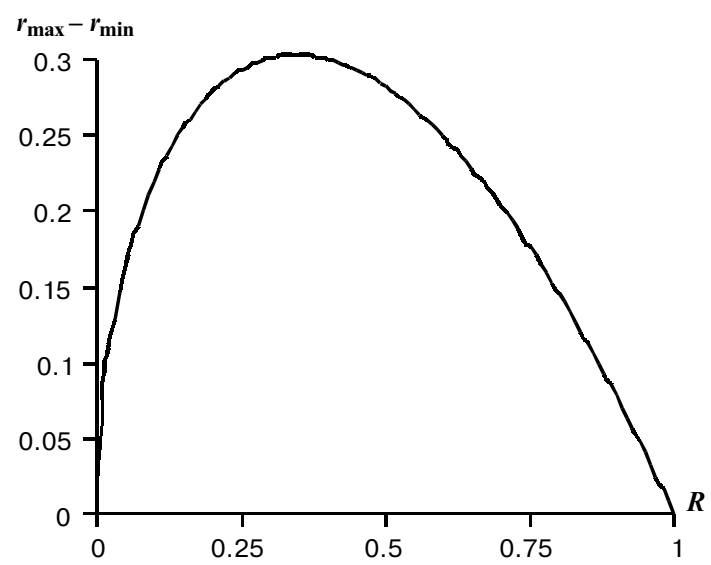

Fig. 3. Dependence of the modulation depth of the mirror reflection coefficient for various values of the reflection coefficient of the sprayed face.

hind the window. Furthermore, to avoid an influence of the interference effects on the radiation spectrum, mirrors of the cavity must be high-reflective (the tight cavity). In this case, the reflection coefficient is described by a weakly-modulated function and practically does not influence the laser radiation spectrum.

One of the $\mathrm{XeCl}$ laser radiation spectrum measured with a tight cavity $\left(R_{1}=R_{2}=99 \%\right)$ under the mixture pressure of $0.3 \mathrm{~atm}$ is presented in Fig. 4 . To verify these lines belonging to a $\mathrm{XeCl}$ molecule, we have measured the spectrum of spontaneous emission. To do this, the cavity mirrors were eliminated and the chamber windows were misaligned. Conditions of the mixture excitation, composition of the mixture, and the common pressure were the same in both the generation and the spontaneous emission cases. A complete correspondence between the generation lines (Fig. 5) and those of the spontaneous emission spectrum is observed, i.e. the fine structure of the generation spectrum under the low pressure is not caused by the cavity effects, instead, it is caused by the energy structure of $\mathrm{XeCl}$ molecules.

In Table 1, the frequencies of $0-3$ transition generation spectrum (Fig. 5) are listed.

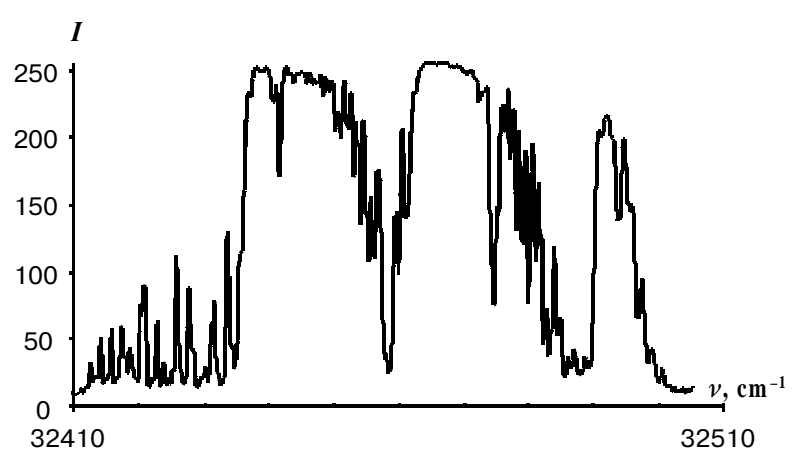

Fig. 4. Emission spectrum of $\mathrm{XeCl}$ laser under the pressure of $0.3 \mathrm{~atm}$.

SQO, 7(3), 2004 


\section{I.V. Blonskyy et al.: On the nature of the fine structure in emission spectra of ...}

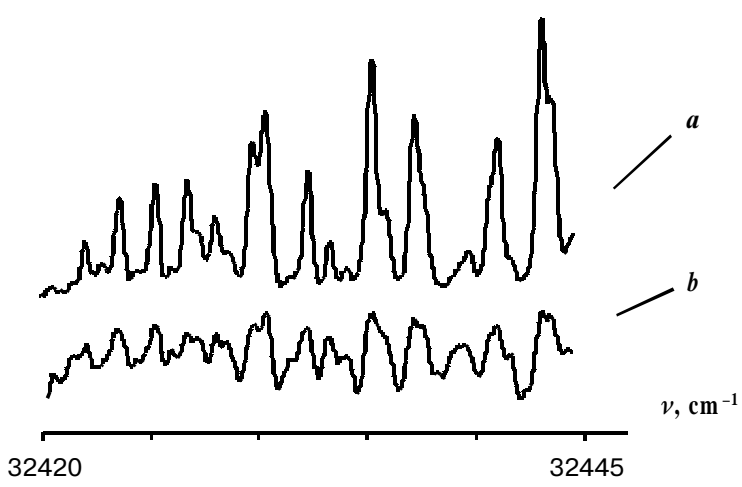

Fig. 5. Spectrum of the induced $(a)$ and spontaneous $(b)$ emission of $\mathrm{XeCl}$ molecules in the region of $0-3$ transition.

The separate rotational transitions can not be observed even with a higher-resolution apparatus is used. According to the selection rules, $P$ and $R$ branches are realized in a rotational spectrum [8]. Furthermore, each of these branches transforms into two branches as a result of the spin splitting.

So, with availability of these four rotational branches, the radiation spectrum is formed by a superposition of many rotational lines. Taking into account that the natural Xe includes 9 isotopes (five of which contribute noticeably), and $\mathrm{Cl}$ includes 2 ones, we can assert that the spectrum observed is formed with superposition of a large number of rotational lines of ten $\mathrm{XeCl}$ molecules possessing different isotopic composition.

If the pressure increases, a gradual widening of the lines takes place and, when pressure achieves $2 \mathrm{~atm}$, tops of only separate peaks are seen on the background of the solid spectrum (see Fig. 1,b).

The presence of the structure caused by the rotation of molecules should be taken into account when the fre-

Table 1. Frequencies of $0-3$ transition generation spectrum.

\begin{tabular}{|c|c|c|}
\hline No & Wavelengths, $\AA$ & Frequencies, $\mathrm{cm}^{-1}$ \\
\hline 1. & 3084.32 & 32412.6 \\
\hline 2. & 3084.24 & 32413.4 \\
\hline 3. & 3084.16 & 32414.2 \\
\hline 4. & 3084.01 & 32415.8 \\
\hline 5. & 3083.87 & 32417.3 \\
\hline 6. & 3083.82 & 32417.8 \\
\hline 7. & 3083.75 & 32418.6 \\
\hline 8. & 3083.58 & 32420.3 \\
\hline 9. & 3083.53 & 32420.9 \\
\hline 10. & 3083.34 & 32422.9 \\
\hline 11. & 3083.24 & 32423.9 \\
\hline 12. & 3083.06 & 32425.8 \\
\hline 13. & 3083.00 & 32426.4 \\
\hline 14. & 3082.87 & 32427.8 \\
\hline 15. & 3082.51 & 32431.6 \\
\hline 16. & 3082.32 & 32433.6 \\
\hline 17. & 3082.27 & 32434.1 \\
\hline
\end{tabular}

$S Q O, 7(3), 2004$ quency is tuned. Authors of works [6,7] have observed a manifestation of the fine structure when tuning the frequency of a high-pressure (3 atm) long-pulse $\mathrm{XeCl}$ laser with $\mathrm{Ne}$ as buffer gas.

In the generation spectrum, we also have observed dips caused by in-cavity absorption of $\mathrm{OH}$ radicals. In particular, deep depressions in the 0-2 transition coincide in their wavelengths with $\mathrm{OH}$ absorption lines quoted in work [5], but full identification of $\mathrm{OH}$ absorption lines was not an object of this work.

\section{Conclusions}

Basing on the experiments carried out and analysis of published data, the following conclusions can be made:

1. Manifestation of the interference pattern in $\mathrm{XeCl}$ laser radiation spectra depends on reflection coefficients of cavity mirrors.

2 . In the case of a tight cavity and low-pressure medium, the fine structure of generation spectra is caused by manifestation of the rotational structure of electronvibration transitions.

3. In the case of high-pressure active medium and if the interference effects are eliminated, the structure of $\mathrm{XeCl}$ laser emission spectra is determined by a shape of the vibration bands and their superposition with the $\mathrm{OH}$ radical absorption lines.

4. To consider this issue in more detail, it is necessary to carry out studies of influence of isotopic composition of the active mixture on the fine structure of $\mathrm{XeCl}$ laser radiation spectra.

\section{Reference}

1. C. Jianwen, F. Shufen, L. Miaohong, Efficient operation of Blumlein-discharge-exited $\mathrm{XeCl}$ laser // Appl. Phys. Lett., 37(10), pp. 883-885 (1980).

2. I.S. Gorban', N.G. Zubrilin, A.S. Kucherov, M.P. Chernomorets, The spectral composition of $\mathrm{XeCl}$-laser radiation with an electron beam pumping // Ukr. Fiz. Zh., 29(4), pp. 596598 (1984).

3. N.G. Basov, I.S. Gorban', V.A. Danilychev, N.G. Zubrilin, M.P. Chernomorets, Rotational-translation resonances in electronic-transition spectra of the $\mathrm{XeCl}$ molecule // Sov. Phys. Dokl., 30(3), pp. 223-224 (1985).

4. V.S. Burakov, A. F. Bokhonov, V. A. Titarchuk, On the Structure of the Electric-Discharge XeCl-Laser Spectrum // Journal of applied spectroscopy, 41(6), pp. 1029-1032 (1984).

5. V.S. Burakov and S.N. Raikov, Intracaviti Laser Spectroscopy: Plasma Diagnostics and Spectral Analisis (Review) // Journal of applied spectroscopy, 69(4), pp. 425-448 (2002).

6. S.V. Efimovskii, A.K. Zhigalkin, Yu.I. Karev, S.V. Kurbasov. Continuous tuning of the emission frequency of long pulses $\mathrm{XeCl}$ lasers in the range 307.0-308.93 nm // Quantum Electronics, 19(6), pp. 525-526 (1992).

7. S.V. Efimovskii, S.V. Kurbasov, A.V. Novichkov, K.K. Pal'chikov. Influence of the parameters of a Fabry-Perot etalon on the output characteristics of a narrov-band long-pulse XeCl laser // Quantum Electronics, 23(7), pp. 615-619 (1996).

8. A.V. Abarenov, V.A. Adamovich, A.A. Deryugin, I.V. Kochetov, V.G. Pevgov. Calculation of emission spectra of eximer molecules with taking into account the bound-bound and bound-free transitions // Preprint IAE-4573/12. Moscow: CNIatominform, 1988 (in Russian), 20 p. 